\title{
Effectiveness of percutaneous needle electrolysis and eccentric exercise in chronic Achilles tendinopathy. A case series
}

\author{
Cerdán Fabregat F.j. ${ }^{1}$ \\ ${ }^{1}$ Centro Médico Florida Portazgo, CE Clinic, Alicante, Spain \\ Rev Fisioter Invasiva 2019;2:127.
}

\begin{abstract}
Keywords

- percutaneous electrolysis

- eccentric achilles tendon exercise

- chronic tendinopathy

Objective The objective of the study was to evaluate percutaneous needle electrolysis (PNE) and exercise with eccentric overload on the tendon body in chronic Achilles tendinopathy.

Material and Methods An experimental study involving seven subjects with a total of seven tendons with a medical diagnosis of chronic tendinopathy on the body of the Achilles tendon. A sample of five men $(71.43 \%)$ and two women $(28.57 \%)$ with a laterality of five right tendons and two left tendons. The mean age was 34 years (SD: $7.22), 42.86 \%$ of the sample was active, practicing regular sports activity $(57.14 \%)$ at the amateur level. All tendons had over six-weeks evolution. Pain was assessed using the visual analogue scale (VAS) and functionality was analyzed using the VISA-A scale, whereas tendon structure was evaluated using musculoskeletal ultrasound. All subjects belonged to the same group and were applied the same treatment; three sessions of PNE, additionally, eccentric exercise was added every 48 hours after each intervention. This involved a total of six eccentric sessions. A seven-day period was allowed between the first and second session of PNE, whereas between the second and third session, 14 days were allowed.

Results In the initial evaluation, the VAS score was 7.14 points (SD: 0.83 ) and the VISA-A scale was 34.86 points (SD: 3.72 ). On the ultrasound examination, the VAS presented changes as a degenerative process. After five weeks, the values on the VAS scale decreased by $75.4 \%$ and on the VISA-A scale they increased by $35.2 \%$.

Conclusions The combined programs of PNE plus eccentric exercises are an effective treatment for chronic tendinopathy of the Achilles tendon body.
\end{abstract}

